\title{
PÉNFIGO FOLIÁCEO VARIEDAD Seborreica: REPORTE DE UN CASO
}

\author{
Arenas Claudia Min SerRato Ingrid ${ }^{2}$ \\ ${ }^{1}$ Dermatóloga, Docente de pregrado y postgrado. Universidad Militar Nueva Granada, \\ Hospital Militar Central Bogotá, Colombia \\ ${ }^{2}$ Residente Dermatología, Universidad Militar Nueva Granada, \\ Hospital Militar Central de Bogotá, Colombia. \\ Correspondencia: iserratop@yahoo.com
}

Recibido: Septiembre 2 de 2013 Aceptado: Diciembre 19 de 2013

\begin{abstract}
Resumen
El pénfigo eritematoso o seborréico, también denominado síndrome de Senear-Usher es la variedad leve y localizada del pénfigo foliáceo, de baja incidencia. La mayor parte de los casos se han reportado en adultos entre la segunda y tercera década de la vida, promedio de 54 años, sin predominio entre razas o sexo. Su etiología se debe a la presencia de anticuerpos anti IgG contra la desmogleina 1 de los queratinocitos de la capa granulosa. Clínicamente se presenta en forma de placas eritematoescamosas o eritematocostrosas bien definidas, de aspecto y distribución seborreica (cara, cuello y tronco), que se exacerban a la exposición solar. Su diagnóstico clínico puede ser difícil, ya que se superpone clínicamente con el lupus eritematoso discoide y la dermatitis seborreica, por lo cual es importante tenerlo en cuenta como diagnóstico diferencial en lesiones infiltradas en dorso nasal y región malar en patrón de alas de mariposa.
\end{abstract}

Se presenta el caso clínico de un paciente con pénfigo foliáceo variedad seborreica una entidad de baja incidencia.

Palabras clave: Pénfigo foliáceo, Sindrome de Senear-Usher, desmogleina 1

\section{PEMPHIGUS FOLIACEUS SEBORRHEIC VARIETY: A CASE REPORT}

\begin{abstract}
Pemphigus erythematosus or seborrheic, also called Senear - Usher syndrome,is a mild, localized variety of pemphigus foliaceus, an entity of low incidence. Most cases have been reported in adults between second and third decades of life, average 54 years, no difference between race or sex. Etiology is due to the presence of IgG antibodies against desmoglein 1 in keratinocytes of the granular layer. Clinically, defined erythematous plaques, seborrheic distribution aspect (face, neck and trunk), which are exacerbated by sun exposure. Clinical diagnosis can be difficult as clinically overlaps with discoid lupus erythematosus and seborrheic dermatitis. So, it is important to be considered as differential diagnosis in infiltrated nasal lesions, dorsum and malar region butterfly pattern.
\end{abstract}

We report a case of pemphigus foliaceus- seborrheic variety, a low incidence entity.

Keywords: Pemphigus foliaceus, Senear-Usher syndrome, desmoglein 1 


\title{
PÉNFIGO FOLIÁCEO VARIEDADE Seborreica: RELATO DE CASO
}

\begin{abstract}
Resumo
O pênfigo eritematoso do tipo seborréico, também chamada de síndrome Senear -Usher é variedade leve e localizada do pênfigo foliáceo, de baixa incidência, a maioria dos casos foram relatados em adultos entre a segunda e terceira década de vida, com idade media de 54 anos, sem predominância entre raças ou sexo. A sua etiologia é devido à presença de anticorpos anti - IgG de desmogleína 1 dos queratinócitos da camada granular. Clinicamente, apresenta-se como placas eritematosas ou eritematocostrosas bem definidos, de aspecto e distribuição seborreica (face, pescoço e tronco), que são agravadas pela exposição ao sol. Seu diagnóstico clínico pode ser difícil, pois se sobrepõe clinicamente com lúpus eritematoso discóide e dermatite seborreica, por isso é importante te-lo em mente como diagnóstico diferencial nas lesões infiltradas no dorso da nariz e região malar com asas de borboleta .
\end{abstract}

Apresenta-se o caso de um paciente com pênfigo foliáceo do tipo seborreico uma entidade de baixa incidência com poucos casos relatados na literatura.

Palavras-chave: Pênfigo foliáceo, Senear-Usher, desmogleína 1

\section{Caso clínico}

Paciente masculino de 39 años, natural y procedente de Moniquirá (Boyacá, Colombia), quien consulta por cuadro clínico de un año de evolución de lesiones que inician como ampollas y placas descamativas, pruriginosas que curan dejando máculas hiperpigmentadas pardo oscuras localizadas en mejillas, cuero cabelludo, pecho y espalda. Las lesiones se exacerban con la exposición solar. Ha recibido múltiples tratamientos incluyendo corticoides tópicos de baja potencia, sin obtener mejoría. Niega antecedentes de importancia.

Al examen físico presenta placas edematosas pardovioláceas con descamación en superficie y maculas violáceas de bordes regulares asimétricos de diámetro variable que se encuentran localizadas en región malar, dorso nasal y cuero cabelludo. En pecho y espalda presenta maculas pardo oscuras de aspecto residual. (Figuras 1 y 2)

Con los hallazgos al examen físico sumado a la historia clínica se realiza una impresión diagnóstica de lupus eritematoso discoide. Se solicita biopsia de piel que reporta epidermis con erosión de la capa córnea y acantolisis superficial que se extiende a la capa granulosa de los infundíbulos, dermis superficial y media con infiltrado linfocitario, algunos eosinófilos, polimorfonucleares y melanófagos. (Figura 3)

Inmunoflourescencia directa reporta $\mathrm{C} 3$ ++, IgG positivo, IgA e IgM negativo. (Figura 4) ANAS y Anti Ro negativos.
Con las características clínicas, histológicas y paraclínicas se realiza diagnóstico depénfigo foliáceo variedad seborreica.

Se inicia tratamiento con aceponato de metilprednisolona crema $0.1 \%$ sin mejoría clínica y aumento de lesiones en mejillas por lo cual se indica tratamiento inmunomodulador con azatioprina $50 \mathrm{mg}$ cada 12 horas con remisión de lesiones cutáneas.

\section{Discusión}

La palabra pénfigo proviene del griego pemphix, que significa ampolla o burbuja. Constituye un grupo de enfermedades de origen autoinmune donde existen anticuerpos dirigidos contra diferentes proteínas de los desmosomas, causando perdida de adhesión de los queratinocitos, produciendo acantolisis y ampollas intraepdermicas $(1,2,3)$. Los pénfigos se clasifican en pénfigo vulgar, pénfigo foliáceo, y pénfigo paraneoplásico, según sus manifestaciones clínicas, histológicas y el tipo de proteínas comprometidas. En el caso del pénfigo foliáceo, segunda variedad más frecuente (30 - 40\% pénfigos) después del pénfigo vulgar, se caracteriza por presentar lesiones solo en piel, anticuerpos anti desmogleina 1 de localización subcorneal; se clasifica en pénfigo foliáceo endémico (fogoselvagem), pénfigo eritematoso y pénfigo herpetiforme $(1,3,4)$.

El pénfigo eritematoso o seborreico, también denominado síndrome de Senear-Usher es la variedad leve y localizada 


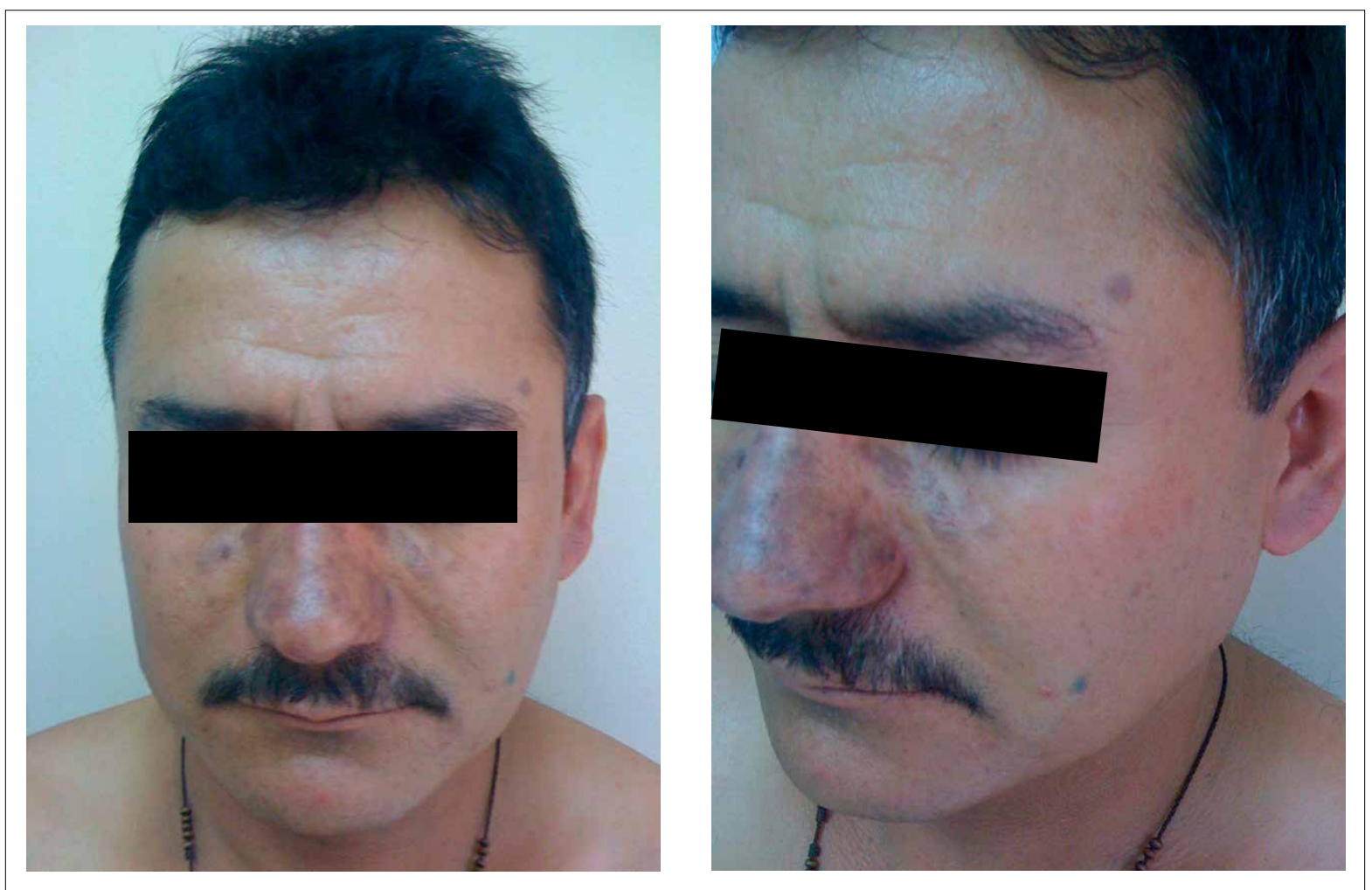

Figuras 1 y 2. Paciente masculino de 39 años que presenta placas edematosas pardovioláceas con descamación en superficie y maculas violáceas de bordes regulares asimétricos de diámetro variable que se encuentran localizadas en región malar, dorso nasal y cuero cabelludo

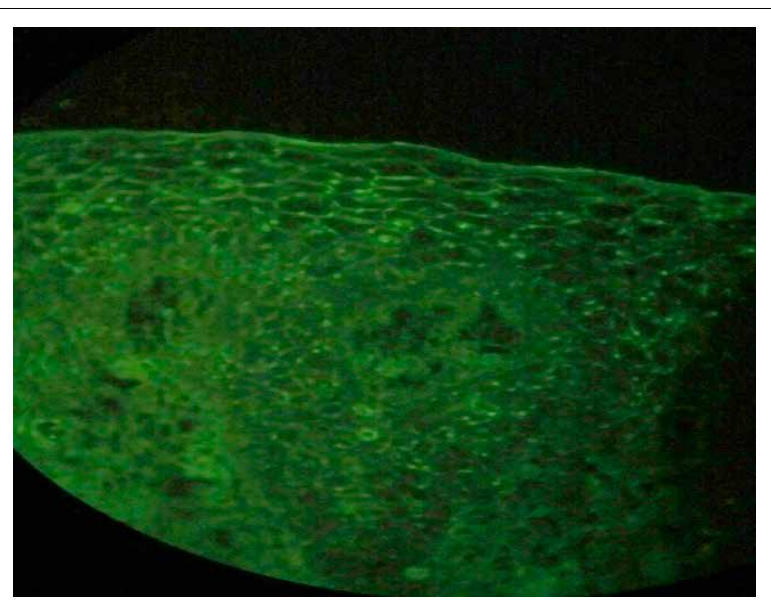

Figura 3. Epidermis con erosión de la capa córnea y acantolisis superficial que se extiende a la capa granulosa de los infundíbulos, dermis superficial y media con infiltrado linfocitario, algunos eosinófilos, polimorfonucleares y melanófagos

del pénfigo foliáceo, con incidencia que oscila entre 0.5 a 3.2 casos por 100.000 habitantes por año, la mayor parte de los casos se han reportado entre los 15-84 años de edad, promedio de 54 años $(2,5)$,en raras ocasiones se

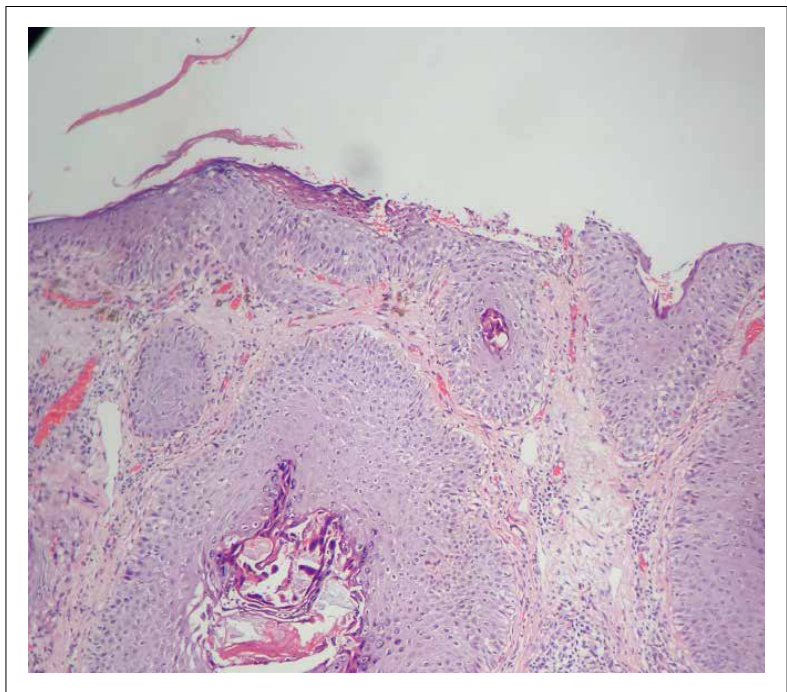

Figura 4. Inmunofluorescencia directa IgG positiva

puede presentar en niños (8), sin predominio entre razas o sexo. El primer caso de pénfigo seborreico fue reportado por Ormsby y Mitchell en 1921, posteriormente Senear y Usher en 1926, describieron un grupo de 11 casos de 
pénfigo con lupus eritematoso. La presencia de acantólisis en el estudio histopatológico confirmó a esta enfermedad como una forma de pénfigo, tomando desde entonces el nombre de síndrome de Senear-Usher. Ormsby en 1933 sugirió el término pénfigo eritematoso y observó que en algunos pacientes permanecía localizado mientras que en otros se diseminaba y evolucionaba hacia pénfigo vulgar o foliáceo. Gray en 1938 fue el primero en considerar al pénfigo eritematoso como una forma localizada de pénfigo foláceo $(9,11)$.

Su etiología se debe a la presencia de anticuerpos anti IgG contra el dominio aminoterminal extracelular de la desmogleina 1 , cadherina desmosomal $160-k D a$, contra queratinocitos de la capa granulosa. Es importante tener en cuenta que la desmogleina 1 se encuentra en toda la epidermis, predominando en piel siendo casi nula en mucosas, pero es en las capas más superficiales donde se expresa en mayor proporción $(3,4,11,17)$.

Clínicamente se presenta en forma de placas eritematoescamosas o eritematocostrosas bien definidas, de aspecto y distribución seborreica (cuero cabelludo, cara, cuello y tronco), que se exacerban a la exposición solar, comprometiendo solo piel no mucosas, pueden estar presentes por meses o años algunos, escasos, con remisión sin tratamiento. En regiones malares adopta un patrón en "alas de mariposa", cuyo diagnóstico clínico puede ser difícil, ya que se superpone clínicamente con el lupus eritematoso discoide y la dermatitis seborreica $(1,2,9,15,18)$.

A la histopatología se encuentra acantolísis en epidermis superficial así como en la capa granulosa, con formación de ampollas subcórneas, en la dermis existe infiltrado moderado, con células inflamatorias a menudo eosinófilos. Acantosis, hiperqueratosis y paraqueratosis pueden aparecer en lesiones antiguas, adicionalmente pueden presentar cambios histopatológicos lupus like (necrosis de queratinocitos por licuefacción o degeneración de la membrana basal). En la inmunofluorescencia directa se observan depósitos de IgG y C3 en toda la epidermis con menor frecuencia predominando solo en capas superiores, también pueden observarse depósitos de IgA e IgM a lo largo de la membrana basal semejando a un patrón en banda del lupus. A la inmunofluorescencia indirecta, ELISA, es posible encontrar hasta en un $90 \%$ anticuerpos anti desmogleina $1 y$ sus títulos son directamente proporcionales al curso de la enfermedad. En algunos casos es posible detectar niveles elevados de ANAS y eosinofilia $(1,2,11,12,14)$.

También se han descrito enfermedades asociadas al pénfigo seborreico:
1) Enfermedades autoinmunes: timoma, miastenia gravis y lupus eritematoso (19-24).

2) Neoplasias: carcinoma broncogénico, adenoma paratiroideo, metástasis hepáticas, epitelioma espinocelular

3) Medicamentos como la penicilamina, propanolol, pyretinol, amoxilina, enalapril, heroína y tiopronina (15, $18,25)$.

El tratamiento varía de acuerdo a la extensión de la enfermedad, las formas localizadas de pénfigo seborreico responden usualmente a esteroides tópicos de alta potencia (en oclusivo de acuerdo la localización y gravedad) o intralesionales y las formas diseminadas a esteroides sistémicos, a dosis de prednisolona de $20-40 \mathrm{mg} /$ día por 1 hasta 30 semanas se han reportado. Sin embargo en aquellos casos que cursan con marcado compromiso de la superficie corporal, que son recidivantes o de difícil manejo se pueden utilizar inmunmoduladores como azatioprina, dapsona, hidroxicloroquina, metrotexate o mofetil micofenolato. En casos muy graves se ha descrito el uso de ciclofosfamida por ciclos junto con dexametasona $(9,15$, $18,26,28)$.

Algunos reportes de caso describen la crioterapia con nitrógeno líquido para casos localizados con muy buena respuesta sin recidivas (18).

\section{Conclusión}

El pénfigo foliáceo variedad seborreica es una entidad de baja incidencia y con pocos casos reportados en la literatura. Es un importante diagnóstico diferencial en lesiones infiltradas en dorso nasal y región malar con patrón de alas de mariposa, donde la biopsia adquiere importancia fundamental para llegar a un diagnóstico definitivo. Es una patología de curso clínico benigno y buen pronóstico.

\section{Referencias}

1 Joly P, Litrowski N. Pemphigus group (vulgaris, vegetans, foliaceus, herpetiformis, brasiliensis). Clinics in Dermatology. 2011; 29: 432-436.

2 Ioannides D, Hytiroglou P, Phelps R, Bystryn JC. Regional variation in the expression of pemphigus foliaceus, pemphigus eritematosus, and pemphigus vulgaris antigens in human skin. J Invest Dermatol.1991; 96(2):159-61.

3 Amagai M, Tsunoda K, Zillikens D, Nagai T, Nishikawa T. The clinical phenotype of pemphigus is defined by the anti-desmoglein autoantibody profile. J Am AcadDermatol1999; 40: 167-170. 
4 Amagai M. Adhesion molecules: I: Keratinocyte-keratinocyte interactions; cadherins and pemphigus. J Invest Dermatol 1999; 104:146-152

5 Amerian ML, Ahmed AR. Pemphigus erythematosus: presentation of four cases and review of literature. J Am AcadDermatol 1984; 10: 215-222.99; 40: 167-170.

6 Kumar K. Incidence of pemphigus in thrissur district, south india. Indian Journal of Dermatology, Venereology and Leprology. 2008;74(4): 349-51.

7 Wieselthier JS, Treloar V, Koh HK, Rao BK, Ahmed AR: Multiple crusted plaques in a woman with systemic lupus erythematosus: pemphigus erythematosus. Arch Dermatol 1991; 127: 1572-1573, 1575-1576.

8 De Peña J, Ramos-Garibay A, Villanueva T. Pénfigo Seborreico Infantil. Rev Cent Dermatol Pascua. 2009; 18(2): 57-60.

9 Medina D, Ramos-Garibay A, Villaseñor P. Pénfigo seborreico facial. Comunicación de un caso. Rev Cent Dermatol Pascua. 2003;12(2):164-167.

10 Steffen C, Thomas D. The Men Behind the Eponym Francis E. Senear, Barney Usher, and the Senear-Usher Syndrome. Am J Dermatopathol. 2003;25:432-436.

11 Nishikawa T, Oshiro A, Takubo H. Inmunological aspects of Senear-Usher syndrome. Keio J Med. 1969;18(2):99-107.

12 Hashimoto T. Recent advances in the study of the pathophysiology of pemphigus. ArchDermatol Res. 2003; 295: 2-11.

13 Jablonska S, Chorzelski T, Blaszczyk M, Maciejewski W. Pathogenesis of pemphigus erythematosus. ArchDermatol Res 1977; 258:135-140.

14 Van Joost T, Stolz E, Blog FB, Ten Kate F, Vuzevski VD. Pemphigus erythematosus:clinical and histo-immunological studies in two unusual cases. ActaDermVenereol1984; 64: 257-260.

15 Peña J, Ramos-Garibay A, Fernández Arista G, Pénfigo eritematoso. Presentación de un caso con manifestación clínica inicial mínima; Rev Cent Dermatol Pascua. 2005;14(3):79-83.

16 Oktarina DA, Poot AM, Kramer D, Diercks GF, Jonkman MF Pas HH. The IgG "Lupus-Band" Deposition Pattern of Pemphigus Erythematosus: Association With the Desmoglein 1 Ectodomain as Revealed by 3 Cases. Arch Dermatol. Jul 16 2012;1-6.
17 Wojnarowska F, Venning VA, Burge SM. Immunobullous diseases. In: Burns DA, Breathnach SM, Cox N, Griffiths CE, Editores. Rook's Textbook of dermatology. Vol. VIII. Malden (MA): Blackwell; 2010. pp. 40.18.

18 Allevato M, Cutrera C, Martínez J. Pénfigo seborreico de localización única en nariz. Tratamiento con criocirugía. Act TerapDermatol. 2007; 30: 250.

19 Megahed M, Goerz G, Kind P, Plewig G. Pemphigus erythematosus with suprabasilaracantholysis and lichenoid tissue reaction or a combination of pemphigus vulgaris and lupus erythematosus: a new entity? Dermatologica 1991; 183: 216-217.

20 Fuxiang, G, Beutner, E. H. Pemphigus erythematosus associated with thymoma: A case report. Cutis. 1999; 64(3), 179.

21 Cruz PD, Coldiron BM, Sontheimer RD. Concurrent features of cutaneous lupus erythematosus and pemphigus erythematosus following myasthenia gravis and thymoma. J Am AcadDermatol. February 1987; 16(2 Pt2):472-480)

22 Ngo AW, Straka C, Fretzin D: Pemphigus erythematosus: a unique association with systemic lupus erythematosus. Cutis 1986; 38: 160-163.

23 Maize JC, Dobson RL, Provost TT: Pemphigus and myasthenia gravis. Arch Dermatol 1975; 111: 1334-1339.

24 Wilkinson SM, Smith AG, Davis MJ, Hollowood K, Dawes PT. Rheumatoid arthritis: an association with pemphigus foliaceous. ActaDermVenereol 1992; 72: 289-291.

25 Alinovi A, Benoldi D, Manganelli P. Pemphigus erythematosus induced by thiopronine. ActaDermatvener (Stockholm) 1982; 62: $452-454$

26 Singh S. Evidence-based treatments for pemphigus vulgaris, pemphigus foliaceus, and bullous pemphigoid: A systematic review. Indian Journal of Dermatology, Venereology and Leprology.2011; 77(4): 456-69.

27 Basset N, Guillot B, Michel B, Meynadier J, Guilhou JJ. Dapsone as initial treatment in superficial Pemphigus. Report of nine cases. Arch Dermatol 1987; 123: 783-5.

28 Beissert S, Werfel T, Frieling U, Bohm M, Sticherling M, Stadler R, et al. A comparison of oral methylprednisolone plus azathioprine or mycophenolatemofetil for the treatment of pemphigus. Arch Dermatol 2006;142:1447-54. 